Rebolj D., Šafarič R., Šorgo A., and Čuš Babič N. (2017). "SMARTCON, Self-MAintaining and RejuvenaTing CONstructions" In: LC3 2017: Volume I - Proceedings of the Joint Conference on Computing in Construction (JC3), July 4-7, 2017, Heraklion, Greece, pp. 553-559. DOI: https://doi.org/10.24928/JC3-2017/0164.

\title{
SMARTCON, SELF-MAINTAINING AND REJUVENATING CONSTRUCTIONS
}

\author{
Danijel Rebolj ${ }^{1}$, Riko Šafarič ${ }^{2}$, Andrej Šorgo $^{3}$, Nenad Čuš Babič ${ }^{4}$
}

\begin{abstract}
Infrastructure systems are established and maintained to satisfy our societal needs for living and transport. The European Roadmap for Cross-Modal Transport Infrastructure Innovation states that by 2030 an improvement of $50 \%$ in infrastructure performance, risk and cost versus a 2010 baseline should be achieved. However, current maintenance methods require intense engagement of highly trained experts and exposure to hazards, they are time consuming and hinder the normal use of constructions. Since infrastructure systems are not able to "care for themselves" they create a heavy burden for society in terms of regular maintenance and total cost of ownership. Therefore, SMARTCON proposes to transform passive constructions into smart structures able to take care of themselves. The envisaged system shall consist of a biomimetic swarm of robots able to perform continuous inspection, analysis of inspected indicators, decision support systems to advise on necessary maintenance or rejuvenation actions and to evaluate implemented actions. The paper is presenting the SMARTCON concept, the preliminary research, and the intended results of a three-year project beginning in early 2017. The project is focusing on bridges as they are considered to be among the most critical infrastructure objects.
\end{abstract}

Keywords: automation, infrastructure, bridges, inspection, maintenance, rejuvenation, robot swarm, biomimetics

\section{INTRODUCTION}

Infrastructure maintenance methods (incl. inspection) require intense engagement of highly trained experts, they are time consuming and hinder the normal use of constructions (Everett et al. 2008). Due to increasing number of constructions and the high maintenance costs (Anon 2012), assuring necessary resources for the normal operation of infrastructure has become a heavy burden for the society. Lack of funding is causing irregularities in maintenance, which is leading to higher repair costs or even deterioration causing constructions to collapse when still in use (Biezma \& Schanack 2007; Deng et al. 2015). Methods have been developed to automate or semi-automate inspection (Oh et al. 2009; Abdel-Qader et al. 2003), recently by using aerial drones (for example the ARIA project financed by NSF (2013) and AEROBI project funded under the H2020 program (2015) - both using LIDAR sensor for bridge inspection). The related

1 Professor, Faculty of Civil Engineering, Transportation Engineering and Architecture, University of Maribor, Maribor, Slovenia, danijel.rebolj@um.si

2 Professor, Faculty of Electrical Engineering and Computer Science, University of Maribor, Maribor, Slovenia, riko.safaric@um.si

3 Professor, Faculty of Natural Sciences and Mathematics, University of Maribor, Maribor, Slovenia, andrej.sorgo@um.si

4 Assistant professor, Faculty of Civil Engineering, Transportation Engineering and Architecture, University of Maribor, Maribor, Slovenia, nenad.babic@um.si 
research efforts known to the project team will provide gradual improvements in terms of speed and quality of traditional procedures, but will not enable a radical improvement, which is essential if we want to achieve an improvement of $50 \%$ in infrastructure performance, risk and cost by 2030 versus 2010 as defined by the ERTRAC-ERRACWaterborne-ACARE-ECTP Task Force (Anon 2013).

The idea of using robots that can climb in and assemble structures in form of a standalone system or swarms is rooted in space construction. The systems developed focused mainly on the assembly of truss-based space structures (Yoshida \& Ueno 1990; Staritz et al. 2001; Doggett 2002). With the advance of the research field, more complex scenarios for construction on earth were tackled, which requires more sophisticated robots due to the presence of gravity and larger variety of structures. Depending on the type of structure to be inspected, many climbing robots have been developed (Balaguer et al. 2006) having different levels of success. These systems were always composed by one robot without interaction with other robotic systems.

Two basic research directions formed over time. One direction builds on swarms of robots that can climb into the structures and manipulate components. The components can be either simple structural elements, e.g. (Nigl et al. 2013), or mechatronic/robotic components, e.g. (Terada \& Murata 2008; Werfel 2012). The other research direction builds on swarms of robots that are both assembly systems and components in one entity, e.g. (Yim et al. 2003). The first approach allows that complexity can be distributed and that, in particular, the components to be assembled are simple and suited for mass production. The second approach has the advantage that only one element has to be designed and coordinated, but critics argue that with the element placed within the structure, the capability of the robot cannot be used actively, and the robot rests, resulting in a kind of idle time. Beyond the mechanical robot systems, (Terada \& Murata 2008) and (Werfel 2012) have developed parametric software to initiate the construction process by simply specifying the shape of the desired structure. Furthermore, open source strategies were tested, allowing a case-based modification and customisation of robots or robot components, e.g. through 3D printing of robot parts (Zykov et al. 2008). None of the mentioned swarm construction approaches was conducted as an integral solution for construction inspection, maintenance and rejuvenation (I-M-R), nor have any of them exceeded the level of a linear, technical approach.

Some ongoing projects show similarities with SMARTCON. In 2014, a three-year NSF founded project started with the goal to establish new design and performance analysis principles, and technologies for the creation of a semi-autonomous network of small mobile robots to aid visual inspection of civil infrastructure, specifically bridges (Martins et al. 2014). The UK funded project on Balancing the impact of City Infrastructure Engineering on Natural systems using Robots (Purnell 2016) has a far more ambitious goal of Zero disruption from Streetworks in UK Cities by 2050. The fiveyear project started in January 2016 with the research goals of autonomous systems for minimally invasive infrastructure sensing, diagnosis and repair, development of advanced robots for deployment in complex live city environments, and the sociotechnical intricacy of the robot - human - natural systems interfaces. The research team intends to develop pioneering robot designs, technical implementations and socioeconomic impact cases linked to specific application requirements, starting with three case-study systems. 


\section{THE SMARTCON CONCEPT}

With SMARTCON, we propose to transform passive constructions into smart structures able to take care of themselves. The envisaged SMARTCON system shall consist of a biomimetic swarm of robots able to perform continuous inspection, analysis of inspected indicators, decision support systems to advise on necessary maintenance or rejuvenation actions and to evaluate implemented actions.

The objective of the SMARTCON project is to develop a completely new concept of "inherent autonomy" of constructions to allow self-maintenance (inspection and repair of existing components) and rejuvenation (replacement of damaged components). The SMARTCON concept is envisaged as a complementing technology for existing and new constructions to prolong their-life time, leading to maximal safety, optimal functionality and minimal costs.

The main objective of SMARTCON is to research and develop appropriate methods and technologies to demonstrate that constructions can become self-maintaining with the aid of a robotic swarm system able to constantly inspect, maintain and rejuvenate (IM-R). Specific objectives:

- Design biomimetic robotic swarm system to enhance constructions for self-I-M-R;

- Establish proper digital model environment with various construction types and robots to enhance design and support simulations;

- Identify and model use case system configuration scenarios;

- Experimentally validate selected use case scenario using digital models and physical models of limited functionality;

- Analyse swarm behaviour and develop software for swarm control;

- Create test and demonstration scenarios and environments;

SMARTCON shall consist of swarms of robots. Each robot (Fig. 1a) shall be capable of:

1. Limited mobility (e.g. crawl, limited climbing),

2. Exchanging extremity parts (e.g. interconnection add-ons, specific type of feet),

3. Connecting with other robots in various configurations for various purposes,

4. Performing as a flexible \& intelligent member (e.g. a rod) of a structural system by modifying its own mechanical characteristics,

5. Communicating with the swarm, receiving and performing instructions, sending data gathered from built-in sensors (e.g. camera),

6. Connecting to electric power source and transfer electric energy through the swarm.

The swarm shall be able to:

1. Form various configurations (either physically connected compositions or robot teams) according to the required task: (a) Moving and carrying (Fig. 1b), (b) Inspection using built-in sensors or specific carried inspection devices (e.g. ultra sound scanner), (c) Maintenance tasks using specific carried equipment (e.g. 3D metal printing device), (d) Structural support by forming into a required truss system (Fig. 1c). 
2. Communicate with the centre to (a) Receive specific instructions, (b) Report on performed tasks, (c) Send required information about the status of the construction.

3. Transfer electric energy from the source node to all members.

In order to assess the concept, we have built in the preliminary research a model of the basic robot to test its elementary functionality (Fig. 1d). The prototype basic two-arm robot is able to detect tie holes in concrete walls and use them to climb, fixing one arm in the tie hole and rotating the other to find the next. It is also able to connect with another basic robot in order to extend the functionality and mobility modes of a robot pair. We have, however, not limited our research to the presented concept, but will explore other possible solutions, e.g. in form of microbots. Effective mobility of the robots on the surface of bridge structures is one of the priorities of the project. Therefore, we plan to research various possibilities of climbing, including the use of Van der Waals force on various surfaces.

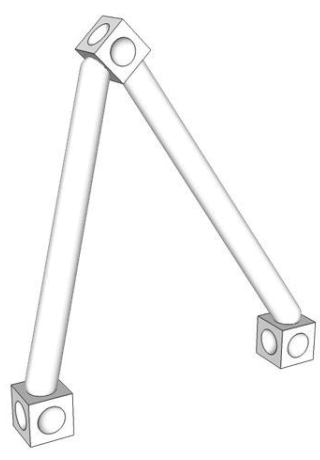

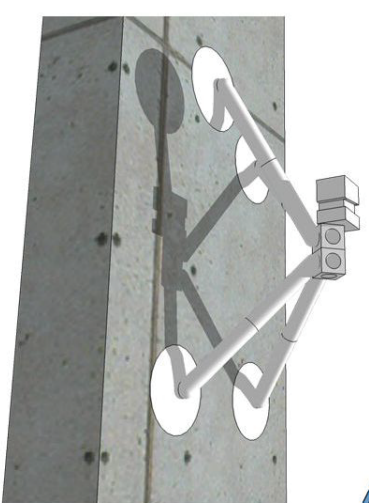

b

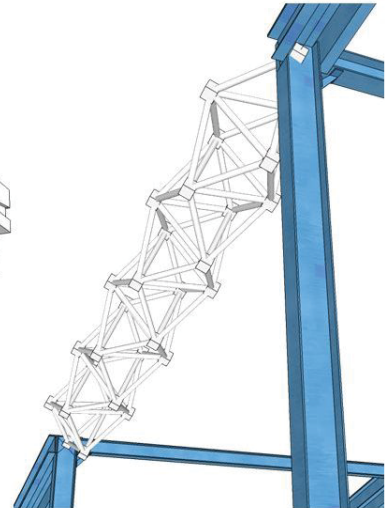

C

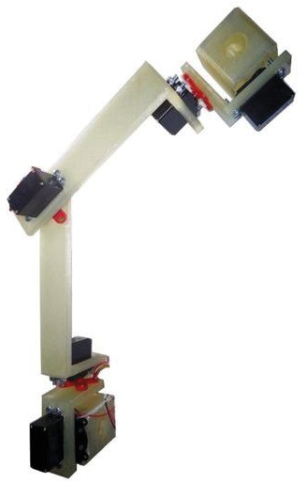

d

Figure 1: A possible design solution of a single robot (a), a simple configuration enabling enhanced mobility and carrying (b), a structural swarm configuration for emergency support (c), and a simple prototype of the basic dual-arm robot (d).

\section{PROJECT PLAN}

The conceptual design of the SMARTCON system is based on the behaviour of vertebrate immune and healing (self-repair) systems. Biological behaviour will be modelled using the unified modelling language (UML) and digital 3D models to better understand and to simulate and thus mimic the successful biological strategies for selfmaintenance. UML will also be used for conceptual SMARTCON system design.

Digital 3D models of constructions and simplified models of robots will be developed to serve for various purposes, including conceptual system design, to better understand deterioration processes, critical locations, materials, and relevant characteristics of constructions, to study navigation methods, locomotion and mobility of robots, and for the experimental purposes. Interoperable 3D design software will be used, which will allow integrated modelling of all necessary characteristics of constructions.

Detailed 3D and kinematic modelling of robots will be used to study their basic functionality, their mechanics, locomotion, design and prototype production. The developed models will be used for simulations of a single robot, robot compositions, swarm behaviour, and of rejuvenation scenarios. Simulations in virtual environments are 
a prospective method to test the concepts and principles of self-I-M-R. Due to the complexity of the system and the limitations of digital models, physical models will be built to simulate and study various aspects of robot behaviour in a real environment. The foreseen problems to be resolved are connected with energy demands, workloads and miniaturization to the working level. For prototype manufacturing, we plan to use 3D printing with various materials.

For mission planning and scheduling of robot swarms we shall use Planning Domain Definition Language (PDDL). To provide maximum flexibility, the control modules will be implemented as a scripting environment, where the user can program the behaviour of virtual agents. This approach will enable the generation of a whole spectrum of agent behaviours, ranging from manual control by the user to fully autonomous behaviour. Series of tests in virtual and physical environment are foreseen during the project to validate and to improve the design of components as well as of the system as a whole.

After the completed system design the strategies and environments for a holistic system test and validation will be designed, considering all partial test environments (virtual and physical) developed in other WPs. The testing environment will also be used as SMARTCON demonstrator. Holistic tests of the prototype system will be run within various spaces of the testing environment (virtual, and physical). According to the results, the system will be optimized and evaluated.

\section{CONCLUSION}

The paper is presenting a new concept of constructions "that can take care of themselves", using biological principles of immune systems. We are aware that the path to achieve a fully functional and reliable intelligent "add-on" for bridges and other types of infrastructure that will allow them to autonomously inspect, maintain and rejuvenate themselves, requires more time and resources then a single, although ambitious project. Each project stage - biomimetic conceptual design, variation and adaptation of robot configurations, grasp planning, and control systems for robot swarm self-organization, intelligent sensorial capacities, fast and flexible charging systems, heterogeneity and decentralized nature of the proposed robotic system - represents a high-risk endeavour. However, it is anticipated that a successful outcome of the project shall pave the way for novel paradigms in a long-term vision of extending existing, as well as creating new structures, able to care for themselves autonomously and thus increase their own safety and quality of service, lower the maintenance costs and prolong their lifetime.

\section{REFERENCES}

Abdel-Qader, I., Abudayyeh, O. and Kelly, M.E. (2003). Analysis of Edge-Detection Techniques for Crack Identification in Bridges. Journal of Computing in Civil Engineering, 17(4), pp.255-263. Available at: http://ascelibrary.org/doi/abs/10.1061/(ASCE)0887-3801(2003)17:4(255) [Accessed November 24, 2016].

Anon. (2012). Demography of railway bridges in Europe, Available at: http://www.pantura-project.eu/Downloads/Appendix 2 to Deliverable Report 5.3 Demography of Railway Bridges in Europe.pdf [Accessed November 24, 2016].

Anon. (2013). Roadmap for cross-modal transport infrastructure innovation - Towards a performing infrastructure. ERTRAC-ERRAC-Waterborne-ACARE-ECTP Task Force, 
p.32. Available at: http://www.ertrac.org/uploads/documentsearch/id2/joint-etp-taskforce-roadmap_84.pdf [Accessed September 27, 2015].

Balaguer, C., Gimenez, A., Huete, A.J., Sabatini, A.M., Topping, M. and Bolmsjo. (2006). The MATS robot: service climbing robot for personal assistance. IEEE Robotics \& Automation Magazine, 13(1).

Biezma, M.V. and Schanack, F., (2007). Collapse of Steel Bridges. Journal of Performance of Constructed Facilities, 21(5), pp.398-405. Available at: http://ascelibrary.org/doi/abs/10.1061/(ASCE)0887-3828(2007)21:5(398).

Deng, L., Wang, W. and Yu, Y. (2015). State-of-the-art review on the causes and mechanisms of bridge collapse. Journal of Performance of Constructed Facilities. Available at: http://dx.doi.org/10.1061/(ASCE)CF.1943-5509.0000731.

Doggett, W. (2002). Robotic assembly of truss structures for space systems and future research plans. In Proceedings, IEEE Aerospace Conference. IEEE, pp. 7-3589-73598. Available at: http://ieeexplore.ieee.org/articleDetails.jsp?arnumber $=1035335$ [Accessed November 24, 2016].

Everett, T.D., Weykamp, P., Capers, H.A., Cox, W.R., Drda, T.S., Hummel, L., Jensen, P., Juntunen, D.A., Kimball, T. and Washer, G.A. (2008). Bridge Evaluation Quality Assurance in Europe, Available at: http://international.fhwa.dot.gov/pubs/pl08016/pl08016.pdf [Accessed November 24, 2016].

Martins, N.M., Bergbreiter, S. and La, R. (2014). NSF Award\#1446785 - CPS: Synergy: Collaborative Research: Designing semi-autonomous networks of miniature robots for inspection of bridges and other large infrastructures. Available at: https://www.nsf.gov/awardsearch/showAward?AWD_ID=1446785 [Accessed March 22, 2017].

Nigl, F., Blum, J.E. and Lipson, H. (2013). Structure-Reconfiguring Robots: Autonomous Truss Reconfiguration and Manipulation. IEEE Robotics \& Automation Magazine, 20(3), pp.60-71. Available at: http://ieeexplore.ieee.org/lpdocs/epic03/wrapper.htm?arnumber $=6547225$ [Accessed November 24, 2016].

Oh, J.-K., Jang, G. Oh, S. Lee, J.H, Yi, B. Moon, Y.S., Lee, J.S and Choi, Y. (2009). Bridge inspection robot system with machine vision. Automation in Construction, 18(7), pp.929-941. Available at: http://www.sciencedirect.com/science/article/pii/S0926580509000600 [Accessed November 24, 2016].

Purnell, P. (2016). Balancing the impact of City Infrastructure Engineering on Natural systems Robots. Aving Avalable http://gow.epsrc.ac.uk/NGBOViewGrant.aspx?GrantRef=EP/N010523/1 [Accessed March 12, 2017].

Staritz, P., Skaff, S., Urmson, C. and Whittaker, W. (2001). Skyworker: a robot for assembly, inspection and maintenance of large scale orbital facilities. In Proceedings of the 2001 IEEE International Conference on Robotics and Automation (ICRA '01). pp. 4180-4185.

Terada, Y. and Murata, S. (2008). Automatic Modular Assembly System and its Distributed Control. The International Journal of Robotics Research, 27(3-4), pp.445462. Available at: http://ijr.sagepub.com/content/27/3-4/445.abstract [Accessed November 24, 2016].

Werfel, J. (2012). Morphogenetic Engineering. In R. Doursat, H. Sayama, and O. Michel, eds. Understanding Complex Systems. Berlin, Heidelberg: Springer Berlin Heidelberg, 
pp. 115-140. Available at: http://www.springerlink.com/index/10.1007/978-3-64233902-8 [Accessed November 24, 2016].

Yim, M., Roufas, K., Duff, D., Zhang, Y., Eldershaw, C. and Homans S. (2003). Modular Reconfigurable Robots in Space Applications. Autonomous Robots, 14(2-3), pp.225237. Available at: http://dl.acm.org/citation.cfm?id=610695.610708 [Accessed November 24, 2016].

Yoshida, T. and Ueno, H. (1990). Development of self- Mobile Space Manipulator system. In Proceedings of the International Symposium on Artificial Intelligence, Robotics and Automation in Space. Kobe, pp. 89-92.

Zykov, V., Williams, P., Lassabe, N. and Lipson H. (2008). Molecubes Extended: Diversifying Capabilities of Open-Source Modular Robotics. In International Conference on Intelligent Robots and Systems (IROS), workshop on SelfReconfigurable Robotic. Nice. Available at: http://citeseerx.ist.psu.edu/viewdoc/download?doi=10.1.1.140.4555\&rep=rep1\&type= pdf [Accessed November 24, 2016]. 